\title{
Sobre la adaptación, aplicación y evaluación de cursos abiertos masivos online de Matemáticas en la plataforma EdX
}

S. Moll, A.J. Guirao, A. Herrero. J.-A. Moraño

Dpto. de Matemática Aplicada, Universitat Politècnica de València, Spain, \{sanmollp,anguisa2,aherrero,jomofer\}@mat.upv.es

\begin{abstract}
In the last years, supported by the Technical University of Valencia, several MOOC's have been developed with the aim of facilitating the learning process and/or expanding the knowledge needed by the students. In this context, with the cooperation of the DMA, we had presented at the UPV [X] platform a collection of four MOOC's titled 'Basic Mathematics: Numbers and Terminology, Derivatives, Integrals and Algebra' covering basic math skills that students need the first year for the different degrees in engineering.

A few months ago the UPV became a member of the EDX network and a major consequence has been to increase the scope of Bases Mathematics courses. This has meant a necessary adaptation of content, a much more progressive approach and improving the evaluation form.

In the article we present the changes made in the process of adaptation to the EDX platform, comparing actual results with the data obtained from previous years and suggesting improvements in the assessment of MOOC's. We have developed a survey with the corresponding tool and an analysis of the opinions of the students is presented.
\end{abstract}

Keywords: MOOC, EdX platform, Mathematica.

\footnotetext{
Resumen

En los últimos años, con el apoyo de la Universidad Politécnica de Valencia, se han desarrollado diversos MOOC's, con el objetivo de facilitar el aprendizaje y/o ampliar los conocimientos que necesitan los estudiantes. En este marco, con la colaboración del DMA, hemos presentado en la plataforma UPV $[X]$ una colección de cuatro MOOC's titulados Bases Matemáticas: Números y Terminología, Derivadas, Integrales y Álgebra que
} 
Sobre la adaptación, aplicación y evaluación de cursos abiertos masivos online de Matemáticas en la plataforma EdX

cubren los conocimientos básicos de matemáticas que los estudiantes necesitan el primer año de los diferentes grados en ingeniería.

Desde hace pocos meses la UPV es miembro de la red EdX y una de las principales consecuencias ha sido el aumento del alcance de los cursos de Bases Matemáticas. Esto ha significado una necesaria adaptación de los contenidos, una metodología mucho más progresiva y una mejora de la forma de evaluación.

En el artículo se presentan las modificaciones realizadas en el proceso de adaptación a la plataforma EdX, y los resultados actuales comparándolos con los datos obtenidos en años anteriores y proponiendo mejoras en la evaluación de los MOOC's. Se ha elaborado un sondeo con la herramienta correspondiente así como un análisis de las opiniones de los estudiantes y de los resultados obtenidos por los mismos.

Palabras clave: MOOC, plataforma EdX, Matemáticas.

\section{Introducción}

Desde hace unos años, en la Universitat Politècnica de València, se vienen desarrollando Cursos Masivos Online Abiertos (MOOC), con el objetivo de facilitar el aprendizaje y complementar los conocimientos que necesitan los estudiantes. El potencial de estos cursos es muy amplio y puede abarcar muchos ámbitos, sobre todo en la comunidad universitaria (Aiken, 2013). Pueden emplearse para introducir nuevos temas, y asignaturas, para profundizar o ampliar conceptos, o como refuerzo de conocimientos previos. Universidades importantes como el Instituto Tecnológico de Massachusetts (MIT) o Harvard han sido los pioneros en este tipo de enseñanzas, con un éxito mundialmente reconocido.

El término MOOC, que es el acrónimo en inglés de massive open online course, fue acuñado por Dave Cormier en 2008, y es una modalidad de educación abierta y a distancia en la que se imparten cursos gratuitos a través de plataformas educativas, normalmente vinculadas con las universidades.

Una de las plataformas educativas online más importantes a nivel mundial es EdX, fundada por las universidades MIT y Harvard en mayo del 2012. Fue creada por estudiantes e instituciones que buscaban adaptarse y mejorar el proceso de aprendizaje empleando la tecnología disponible, una metodología educativa innovadora y cursos rigurosos sobre diferentes temas. Hoy en día más de 78 universidades de todo el mundo colaboran en esta plataforma ofreciendo cursos online de diferente nivel, aunque predominando los de nivel 
universitario en un amplio rango de disciplinas. En EdX también se realiza investigación educativa basada en la forma en cómo se emplea la plataforma para mejorar el aprendizaje de los estudiantes. Cabe destacar que EdX ha sido creada sin ánimo de lucro y se basa en software de código abierto.

En este marco, y gracias a la colaboración del DMA, hemos presentado en la plataforma creada por la UPV, UPV[X], una colección de cuatro MOOC's titulados Bases Matemáticas: Números y Terminología, Derivadas, Integrales y Álgebra que cubren los conocimientos básicos de matemáticas que los estudiantes necesitan el primer año de los diferentes grados en ingeniería. Los objetivos que se perseguían eran:

- equiparar el nivel de los estudiantes de primer año de los diferentes grados en ingeniería en matemáticas

- mejorar la motivación y percepción de las asignaturas de matemáticas por parte de los estudiantes

- evitar el abandono

- lograr una más rápida y natural adquisición de las competencias específicas de las asignaturas involucradas

Sin embargo desde hace pocos meses la UPV es miembro colaborador de la plataforma EdX y como consecuencia ha aumentado el alcance de los cursos de Bases Matemáticas. Esto ha significado una necesaria adaptación de los contenidos, una metodología mucho más progresiva y una mejora de la forma de evaluación. En el artículo se presentan las modificaciones realizadas en el proceso de adaptación a la plataforma EdX, los resultados obtenidos y la opinión de los participantes.

\section{Objetivos}

Los objetivos que se plantean en el artículo son:

- Adaptar los cursos MOOC's de Matemáticas Básicas (Números y Terminología, Derivadas, Integrales y Álgebra) a la nueva plataforma EdX.

- Mejorar la metodología de forma que se adecue a un público más amplio y variado. Ésta ha de ser más progresiva, detallando con rigor los objetivos, los elementos de aprendizaje y las evaluaciones.

- Reducir la duración de los vídeos cuando sea posible, para favorecer la fluidez del aprendizaje. 
Sobre la adaptación, aplicación y evaluación de cursos abiertos masivos online de Matemáticas en la plataforma EdX

- Estudiar una forma de evaluación que se adapte mejor a los diferentes orígenes de los participantes.

- Evaluar los resultados académicos, el seguimiento y las opiniones de los participantes de los cursos.

\section{Combinando conceptos, problemas y simulaciones}

Partiendo de los cursos de Bases Matemáticas que se realizaron para la plataforma UPV[X], se han realizado modificaciones significativas en cuanto al contenido y la evaluación de forma que se puedan adaptar mejor a un mayor número de usuarios (Kellogg, 2013).

Para las modificaciones se ha empleado la plataforma de EdX y las herramientas de edición con las que cuenta (EdX Studio).

Para adaptarnos a la nueva plataforma se han realizado los siguientes cambios:

- Lenguaje: Se ha intentado usar un lenguaje lo más neutro posible, revisando los textos aportados, así como la redacción de las cuestiones y problemas planteados a los estudiantes/usuarios de la plataforma.

- Contenido: En cuanto al contenido se ha tratado de optimizar los tiempos de los videos al máximo, intentando que las unidades estuvieran lo más atomizadas posible de forma que tengan sentido individualmente y, a su vez, en el conjunto de unidades. La duración de los vídeos tiene una media de 8 minutos y esta duración está pensada para facilitar el aprendizaje, evitando el abandono en la medida de lo posible.

Por otra parte, se ha tratado de mejorar la parte didáctica en las presentaciones, aumentando el número de ejemplos, y haciendo una revisión de las explicaciones teóricas (King, 2014). También se ha reestructurado las unidades y la presentación de las mismas para hacerlas más atractivas y lograr mejorar la motivación y la percepción de la estructura del proceso de aprendizaje. Además se ha organizado el curso con especial cuidado en la secuencia de unidades, de forma que el aprendizaje sea lo más progresivo posible. Respecto a este aspecto cabe mencionar que cuando el público objetivo de los cursos era principalmente los alumnos de nuevo ingreso en las escuelas de ingeniería de la UPV, se partía de una base común en cuanto al conocimiento del que venían los estudiantes, ya que para acceder a los estudios universitarios es necesario pasar una prueba de nivel. A pesar de que no todos los alumnos poseían las capacidades y habilidades matemáticas necesarias para afrontar con éxito las asignaturas de matemáticas de los primeros cursos, y existía una gran variabilidad al respecto, sí que había una consciencia común de lo que se debía saber. Al aumentar el público objetivo de forma exponencial, el orden en la exposición de los contenidos es 
fundamental para construir un conocimiento progresivo y afianzado en las unidades previas.

Por último, se ha tratado de mejorar la participación activa en el foro para detectar carencias y debilidades en los conocimientos de los participantes y reforzar los conceptos que sean necesarios. A este respecto, se ha proporcionado material adicional y referencias de utilidad a los participantes de forma general, y puntual, cuando ha sido necesario, por ejemplo cuando existía una duda generalizada en el foro.

- Evaluación: Se ha revisado con detalle la evaluación realizada en cada unidad semanal y en las pruebas finales. Se ha intentado que las preguntas estuvieran en orden creciente de dificultad, de forma que se mantenga la motivación del estudiante. La dificultad final ha procurado ser lo suficiente para suponer un reto al estudiante, pero que este reto fuera alcanzable con un determinado esfuerzo.

Lo mismo se ha realizado con las evaluaciones a lo largo del curso intentando que en estas se repasen los conceptos básicos de las unidades anteriores de forma continua. El objetivo ha sido que al aplicar los conceptos aprendidos repetidamente en diferentes situaciones el alumno asiente los conocimientos básicos que se intentan comprender.

- Opiniones: De la experiencia de los MOOC's anteriores se obtuvieron encuestas de opinión sobre los cursos. En esta ocasión también se han realizado encuestas a los estudiantes para determinar el éxito o fracaso de las modificaciones realizadas. Los autores, como sondeo han ofertado las unidades que conforman los cursos, como parte de un método de refuerzo en las asignaturas impartidas en los grados de Ingeniería Biomédica, Ingeniería de Tecnología Industrial, Ingeniería Aeroespacial e Ingeniería Electrónica. En todos los casos se ponía a disposición de los estudiantes el enlace de la plataforma EdX con los cursos ofertados o bien, cuando no estaba disponible el MOOC, los enlaces de los polimedias, que conforman las unidades atomizadas de los unidades, con el doble fin de que los estudiantes dispongan de una método para reforzar competencias y conocimientos y, por otra parte, evalúen de forma crítica los objetos de aprendizaje para su mejora. Con el fin de recabar datos se les entregó una encuesta relativa a estos cursos, y los resultados de estas encuestas se presentan en este artículo.

\section{Resultados}

Expondremos en esta unidad los resultados obtenidos en cada uno de los cursos de Bases Matemáticas. Los resultados obtenidos son bastante similares para los cuatro cursos, aunque no se dispone de todos los datos referentes al curso de integrales, pues actualmente está todavía en activo.

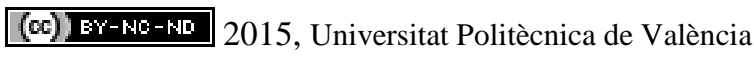

Congreso In-Red (2015) 
Sobre la adaptación, aplicación y evaluación de cursos abiertos masivos online de Matemáticas en la plataforma EdX

\subsection{Bases Matemáticas: Derivadas}

El número de participantes que acabó el curso fue de 3002, constituido por un grupo muy heterogéneo de diferentes procedencias y edades. Como se puede ver en la Fig. 1 la distribución de las edades de los usuarios es bastante dispersa, aunque se aprecia mayor densidad de población en el rango comprendido entre 18 y 32. La edad media ha sido de 27 años.

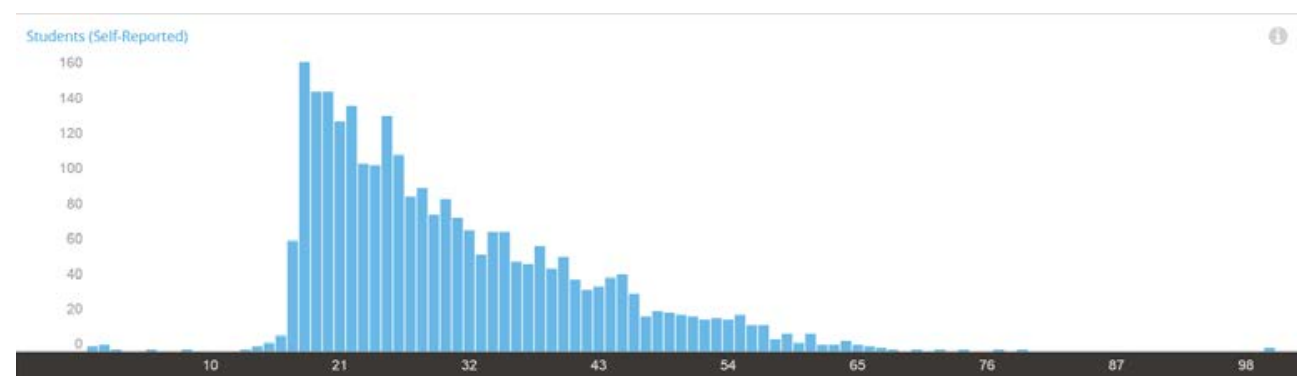

Fig 1. Distribución de la edad de los participantes

De los datos que han aportado los participantes se puede deducir que el 42,8\% de tiene una edad inferior a 25 años, un 34,5\% tiene una edad entre 26 y 40 años y un 18,6\% son mayores de 41 años.

Además de la diferencia de edad de los participantes, los estudios previos también son muy diversos. Cabe destacar que la mayoría de participantes tenían un nivel de estudios correspondientes a Secundaria (31,1\%), a estudios universitarios $(29,1 \%)$ y a máster (22,3\%) como se puede ver en la Fig. 2.

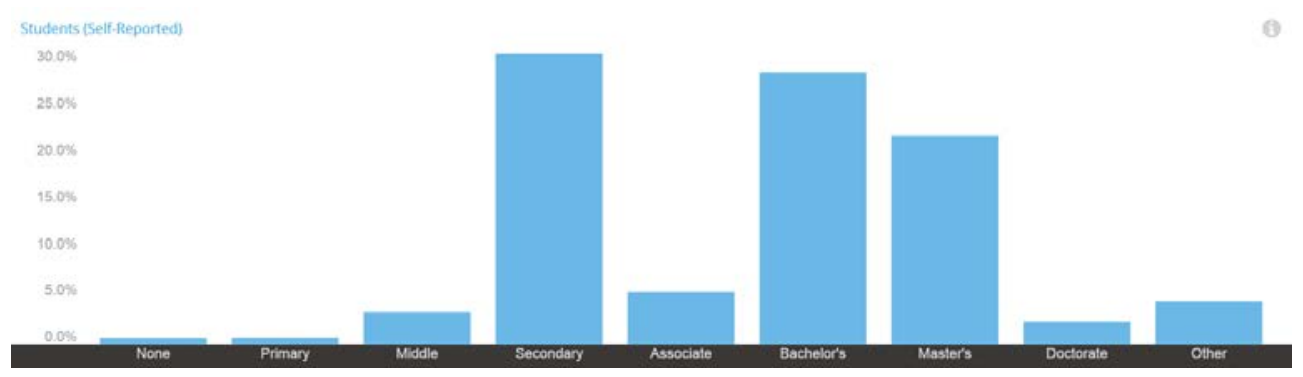

Fig 2. Nivel de estudios de los participantes

Por último, la distribución geográfica de los participantes también ha sido muy variada. Como era de esperar, en los países de habla hispana se concentran la mayoría de los participantes. España ha sido el país que más participantes ha aportado (21\%), seguida por 
México (18\%) y Colombia (12\%). En total, el curso ha tenido participantes de 82 países. Ver Fig. 3.

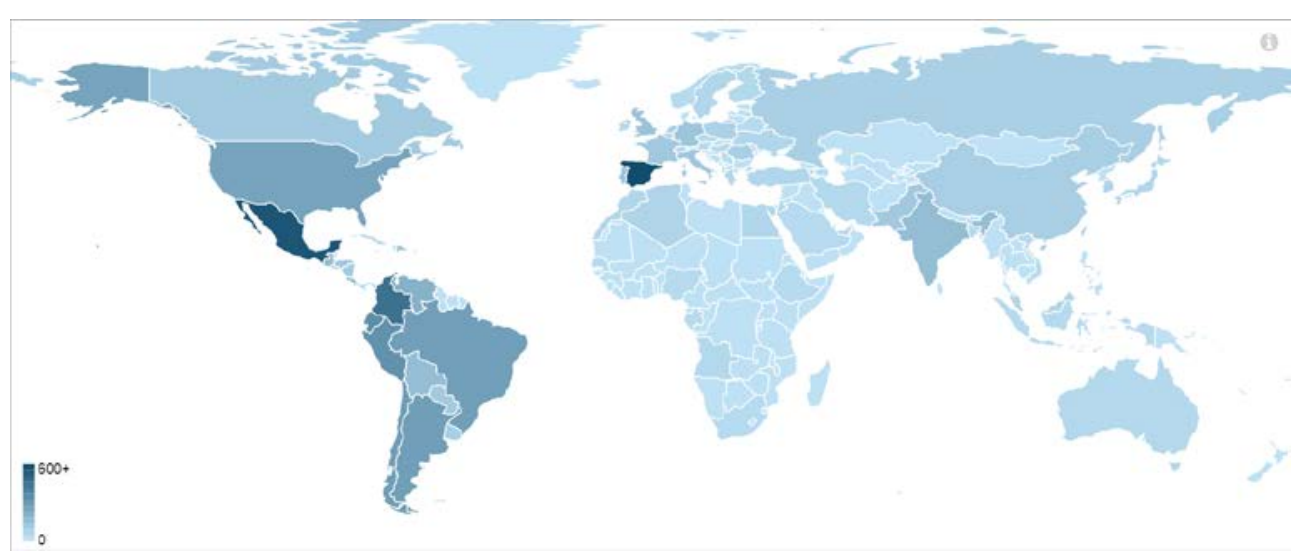

Fig 3. Distribución geográfica de los participantes

Para más detalles de la distribución geográfica de los participantes, en la Tabla 1 se recogen las participaciones por países de los más representativos.

Tabla 1. Distribución Geográfica

\begin{tabular}{lcc} 
País & Porcentaje & Total Participantes \\
\hline España & 20.7 & 621 \\
\hline México & 18.2 & 547 \\
\hline Colombia & 11.7 & 351 \\
\hline Ecuador & 6.4 & 191 \\
\hline Perú & 5.9 & 176 \\
\hline Chile & 4.3 & 128 \\
\hline Argentina & 4.1 & 123 \\
\hline Brasil & 4.1 & 122 \\
\hline Estados Unidos & 3.5 & 106 \\
\hline Venezuela & 2.1 & 63
\end{tabular}

En lo que respecta al género de los participantes, el 74,4\% de los mismos son de género masculino y el $25,3 \%$ son de género femenino.

El curso de Bases Matemáticas Derivadas está dividido en 5 unidades: Concepto de función tipos y propiedades, Definición de Derivada, Cálculo de Derivadas, Optimización y Máximos y Mínimos condicionados. En la Fig. 4 se muestran los porcentajes obtenidos de

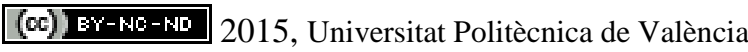


Sobre la adaptación, aplicación y evaluación de cursos abiertos masivos online de Matemáticas en la plataforma EdX

éxito en la evaluación de cada unidad. Como se puede apreciar, en todas las unidades, más del $84 \%$ de participantes han superado las unidades con éxito. Las evaluaciones han mantenido un nivel de dificultad creciente, y esto se ha visto reflejado en las evaluaciones: el porcentaje de aprobados ha descendido desde la unidad 2 (la primera que está dedicada a derivadas) hasta la unidad 5. Sin embargo, la evaluación final ha incrementado el porcentaje hasta un $88,8 \%$.

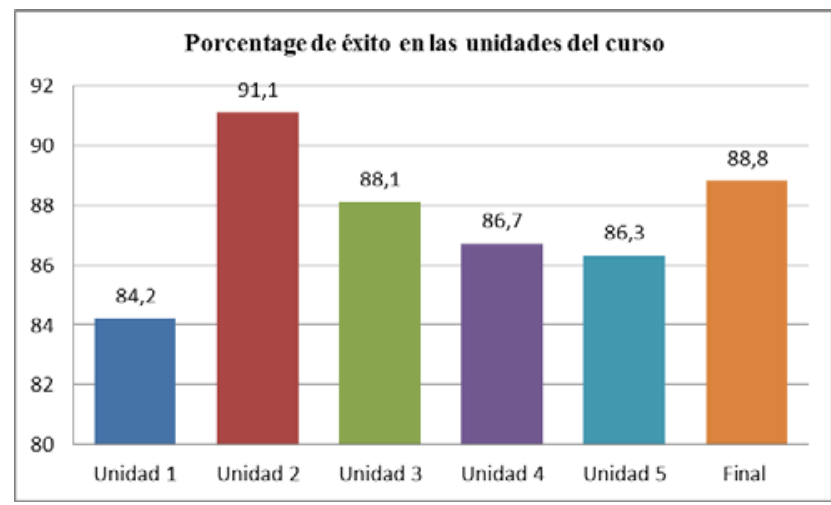

Fig 4. Tasa de éxito de cada unidad del curso Derivadas

\subsection{Bases Matemáticas: Números y Terminología}

El número de participantes fue de 2286. Como en el curso de derivadas, estaba constituido por un grupo heterogéneo de diferentes procedencias y edades. Como se puede ver en la Fig. 5 la distribución de las edades muestra mayor densidad en el rango comprendido entre 18 y 43. La edad media ha sido de 29 años.

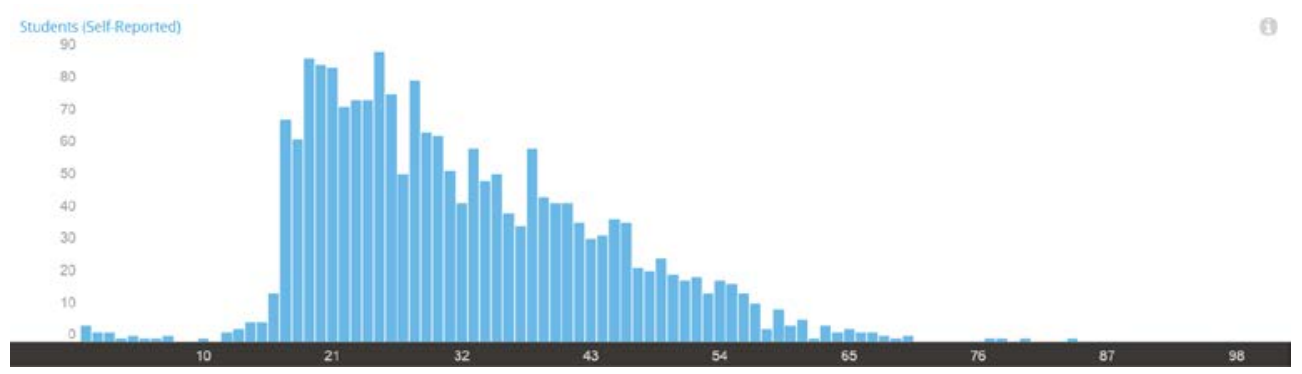

Fig 5. Distribución de la edad de los participantes

De los datos obtenidos sabemos que el 36,7\% tiene una edad inferior a 25 años, un 36,1\% tiene una edad entre 26 y 40 años y un 23,5\% son mayores de 41 años. 
Como en el caso de Derivadas el nivel de estudios de los participantes es muy variado aunque la mayoría de participantes tenían un nivel de estudios correspondientes a Secundaria (31\%), a estudios universitarios (26.8\%) y a máster $(23,2 \%)$ como se puede ver en la Fig. 6.

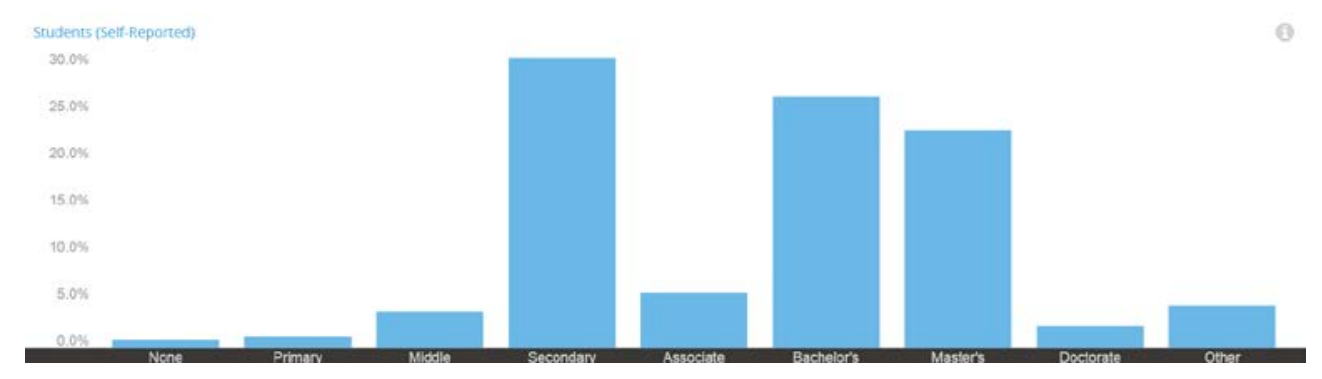

Fig 6. Nivel de estudios de los participantes del curso de Números y Terminología

En cuanto a la distribución geográfica de los participantes se ha obtenido un porcentaje más alto en España que en el curso de Derivadas y menor participación de los países de habla hispánica. Se pueden obtener más detalles de la distribución en la Tabla 2.

Tabla 2. Distribución Geográfica Números y Terminología

\begin{tabular}{llc} 
País & Porcentaje & Total Participante: \\
\hline Spain & 23.6 & 540 \\
\hline Mexico & 15.9 & 364 \\
\hline Colombia & 11.5 & 264 \\
\hline Chile & 7.5 & 172 \\
\hline United States & 6.0 & 138 \\
\hline Peru & 5.0 & 114 \\
\hline Ecuador & 3.6 & 82 \\
\hline Argentina & 3.5 & 80 \\
\hline Brazil & 2.8 & 65 \\
\hline Venezuela & 2.1 & 47
\end{tabular}

De los participantes el 70\% de los mismos son de género masculino y el 30\% son de género femenino.

El curso de Bases Matemáticas Números y Terminología está dividido en 3 unidades: Terminología y Conceptos Básicos, Conjuntos Numéricos y Números Complejos. En la Fig. 7 se muestran los porcentajes de éxito obtenidos en la evaluación de cada unidad. En este caso, los porcentajes son más elevados, aunque esto puede ser debido al menor nivel de

\section{(c)) EY-NC-ND 2015, Universitat Politècnica de València}


Sobre la adaptación, aplicación y evaluación de cursos abiertos masivos online de Matemáticas en la plataforma EdX

dificultad de los conceptos evaluados. La unidad con menor tasa de aprobados ha sido la parte de números complejos.

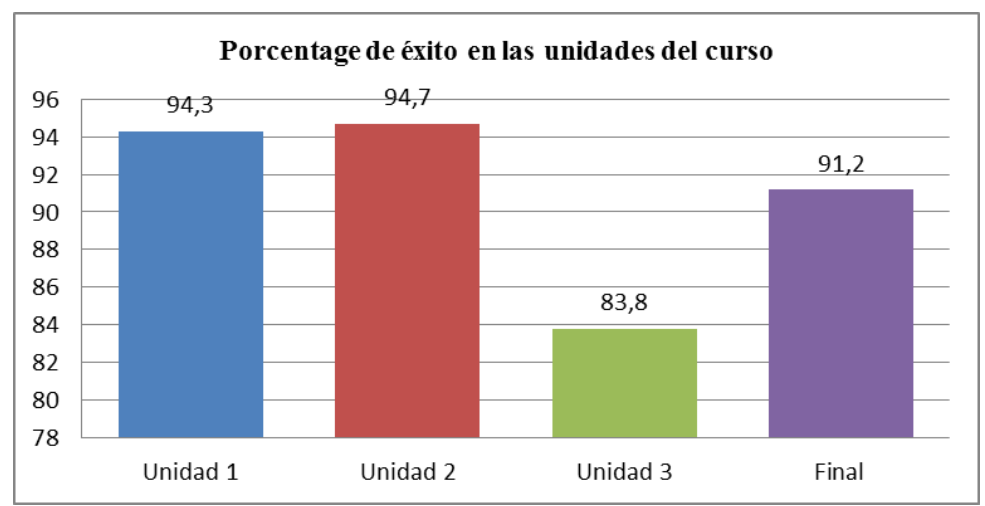

Fig 7. Tasa de éxito de cada unidad del curso Números y Terminología

\subsection{Bases Matemáticas: Álgebra}

El número de participantes de este curso 3385. Como en los cursos anteriores los participantes forman un grupo heterogéneo de diferentes procedencias y edades. Como se puede ver en la Fig. 8 la distribución de las edades muestra mayor densidad en el rango comprendido entre 18 y 32. La edad media ha sido de 27 años.

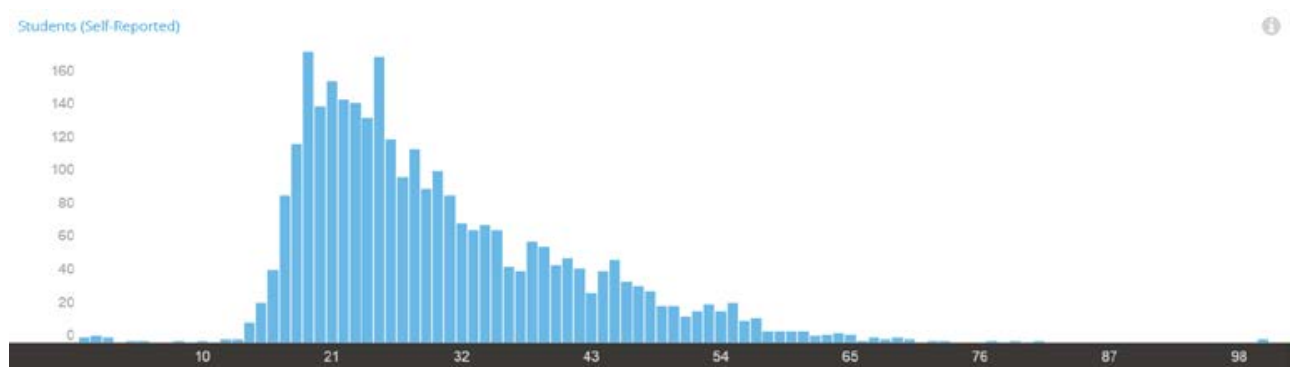

Fig 8. Distribución de la edad de los participantes en el curso de Álgebra

De los datos obtenidos sabemos que el 44,6\% tiene una edad inferior a 25 años, un 33,4\% tiene una edad entre 26 y 40 años y un $18 \%$ son mayores de 41 años.

El nivel de estudios de los participantes es muy variado aunque como en los casos anteriores la mayoría corresponden a Secundaria (33,8\%), a estudios universitarios $(28,4 \%)$ y a máster (20,1\%) como se puede ver en la Fig. 9. 


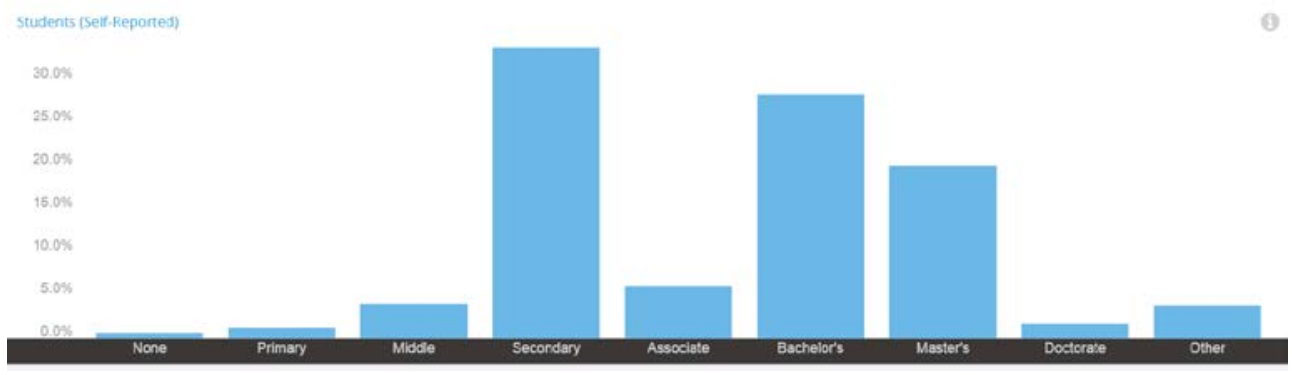

Fig 9. Distribución de la edad de los participantes en el curso de Álgebra

La distribución geográfica de los participantes en el curso de Álgebra se muestra en la Tabla3.

Tabla 3. Distribución geográfica de Álgebra

\begin{tabular}{llc} 
País & Porcentaje & Total Participante: \\
\hline Spain & 21.7 & 732 \\
\hline Mexico & 18.5 & 626 \\
\hline Colombia & 10.7 & 363 \\
\hline United States & 5.3 & 180 \\
\hline Peru & 4.8 & 162 \\
\hline Chile & 4.1 & 138 \\
\hline Argentina & 3.7 & 126 \\
\hline Brazil & 3.0 & 100 \\
\hline Ecuador & 2.9 & 98 \\
\hline India & 1.9 & 65
\end{tabular}

El curso de Bases Matemáticas Álgebra está dividido en 3 unidades. En la Fig. 10 se muestran los porcentajes de éxito obtenidos en la evaluación de cada unidad.

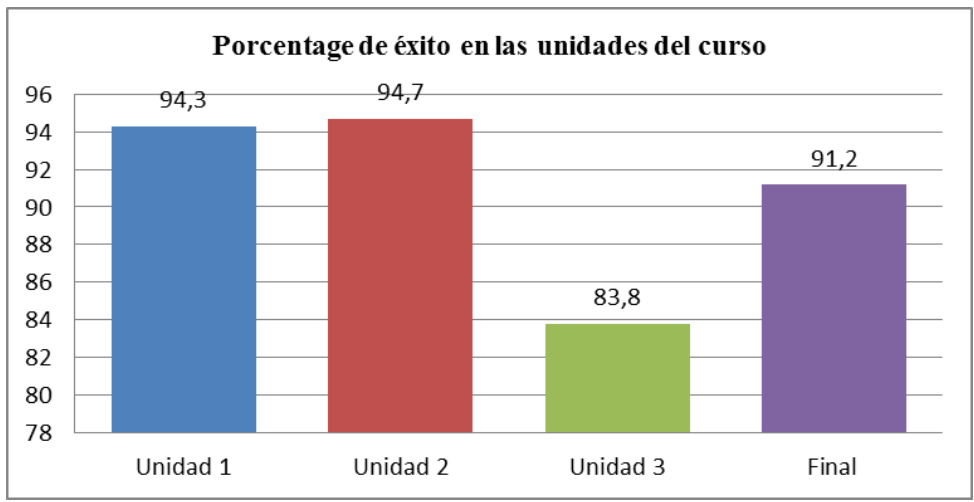

Fig 10. Porcentajes de éxito encada unidad del curso de Álgebra

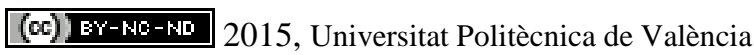

Congreso In-Red (2015) 
Sobre la adaptación, aplicación y evaluación de cursos abiertos masivos online de Matemáticas en la plataforma EdX

\subsection{Bases Matemáticas: Integrales}

Para el curso de Bases Matemáticas Integrales se han obtenido unos datos muy similares a los obtenidos en los otros cursos, excepto por las tasas de éxito que aún no están disponibles.

\subsection{Encuestas}

Se ha pasado una encuesta a 50 estudiantes de los cursos Derivadas e Integrales con el fin de obtener su opinión sobre diferentes aspectos de los cursos. A continuación se presentan algunos de los resultados obtenidos.

Tabla 4: Percepción de los MOOC’s en general

\begin{tabular}{|l|c|}
\hline $\begin{array}{l}\text { ¿Te ha sido de utilidad haber cursado } \\
\text { los MOOC? }\end{array}$ & Respuestas \\
\hline Muy Poco & $0 \%$ \\
\hline Poco & $10,9 \%$ \\
\hline Indiferente & $9 \%$ \\
\hline Bastante & $60,5 \%$ \\
\hline Mucho & $19,6 \%$ \\
\hline N/D & $0 \%$ \\
\hline
\end{tabular}

Una de las modificaciones que se han realizado ha sido la de la evaluación de las sesiones, a este respecto, los estudiantes encuestados nos han proporcionado los datos mostrados en la Tabla 5.

Tabla 5: Evaluación de las sesiones

\begin{tabular}{|l|c|}
\hline $\begin{array}{l}\text { ¿Las evaluaciones son adecuadas para } \\
\text { los contenidos impartidos en el MOOC? }\end{array}$ & Respuestas \\
\hline Muy Poco & $0 \%$ \\
\hline Poco & $15,8 \%$ \\
\hline Indiferente & $27 \%$ \\
\hline Bastante & $57,2 \%$ \\
\hline
\end{tabular}

(c)) EY-NC-ND 2015, Universitat Politècnica de València Congreso IN-RED (2015) 
A continuación se muestran algunas de las cuestiones relativas a los contenidos y el lenguaje y sobre la valoración de los contenidos adicionales.

Tabla 6: Sobre los contenidos

\begin{tabular}{|l|c|}
\hline $\begin{array}{l}\text { ¿El lenguaje empleado y las } \\
\text { explicaciones mostradas te han } \\
\text { resultado fáciles de comprender? }\end{array}$ & Respuestas \\
\hline Muy Poco & $0 \%$ \\
\hline Poco & $8 \%$ \\
\hline Indiferente & $20 \%$ \\
\hline Bastante & $52,4 \%$ \\
\hline Mucho & $19,6 \%$ \\
\hline N/D & $0 \%$ \\
\hline
\end{tabular}

Tabla 7: Material adicional

\begin{tabular}{|l|c|}
\hline $\begin{array}{c}\text { ¿Te han resultados útiles los } \\
\text { materiales } \\
\text { proporcionados? }\end{array}$ & Respuestas \\
\hline Muy Poco & $0 \%$ \\
\hline Poco & $2 \%$ \\
\hline Indiferente & $17 \%$ \\
\hline Bastante & $12,8 \%$ \\
\hline Mucho & $68,2 \%$ \\
\hline N/D & $0 \%$ \\
\hline
\end{tabular}

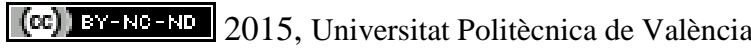


Sobre la adaptación, aplicación y evaluación de cursos abiertos masivos online de Matemáticas en la plataforma EdX

\section{Conclusión}

La incorporación de la UPV como universidad colaboradora en la plataforma EdX ha implicado un aumento muy significativo de los participantes en los cursos de Matemáticas Básicas: Números y Terminología, Álgebra, Derivadas e Integrales, dos de los cuales ya habían sido ofertados en la plataforma UPV[X]. Además del aumento en número, el hecho de que los participantes provengan de diferentes países ha supuesto un reto para estandarizar los cursos de forma que sean asequibles para la mayoría de los usuarios.

Los cambios realizados en los cursos en relación a la evaluación, los contenidos, material adicional y el aprendizaje progresivo han proporcionado unos muy buenos resultados en cuanto al seguimiento de los cursos y las evaluaciones obtenidas. Además, la opinión expresada por los alumnos encuestados indica una alta valoración de los aspectos modificados.

Se han detectado todavía unidades con un porcentaje de éxito más bajo de lo esperado, por lo que cabría revisar tanto la dificultad como el orden de la exposición de los contenidos con el fin de optimizar el proceso de aprendizaje de los participantes en los cursos.

Los porcentajes de éxito han sido más elevados que en las convocatorias anteriores a pesar de que el número de alumnos de esta plataforma quintuplica al de los alumnos en la plataforma UPV[X].

\section{Agradecimientos}

Los autores agradecen al Departamento de Matemática Aplicada de la UPV por el establecimiento de la Ayuda a Proyectos de Innovación Docente, PID-DMA-2014, que financia esta investigación y a la ETSID por su apoyo continuo en los proyectos de innovación docente y.

\section{Referencias}

AIKEN, J.M., et al. (2013). “The Initial State of Students Taking an Introductory Physics MOOC”, PERC Proceedings, Cornwell University, p. 295-309.

KELLOGG, S. (2013). “Online learning: how to make a MOOC”, Nature, vol. 499, p. 369-371. KING, C., ROBINSON, A., VICKERS, J. (2014). “Online education: Targeted MOOC captivates students”, Nature, vol. 505.

UPV[X]. (2015). UPV[X] plataforma. <http://www.upvx.es> [Consulta: 10 de mayo de 2015]

EDX. (2015). Edx Plataform. < https://www.edx.org/> [Consulta: 29 de mayo de 2015] 